\section{Gender Differences in Depression}

\section{Susan Nolen-Hoeksema ${ }^{1}$}

Department of Psychology, University of Michigan, Ann Arbor, Michigan

Abstract
From early adolescence
through adulthood, women are
twice as likely as men to experi-
ence depression. Many differ-
ent explanations for this gender
difference in depression have
been offered, but none seems to
fully explain it. Recent research
has focused on gender differ-
ences in stress responses, and
in exposure to certain stressors.
I review this research and de-
scribe how gender differences
in stress experiences and stress
reactivity may interact to create
women's greater vulnerability
to depression.
Keywords
gender; depression; stress

Across many nations, cultures, and ethnicities, women are about twice as likely as men to develop depression (Nolen-Hoeksema, 1990; Weissman et al., 1996). This is true whether depression is indexed as a diagnosed mental disorder or as subclinical symptoms. Diagnosable depressive disorders are extraordinarily common in women, who have a lifetime prevalence for major depressive disorder of $21.3 \%$, compared with $12.7 \%$ in men (Kessler, McGonagle, Swartz, Blazer, \& Nelson, 1993).

Most explanations for the gender difference in depression have focused on individual variables, and studies have attempted to show that one variable is better than another in explaining the difference. In three decades of research, however, no one variable has single-handedly accounted for the gender difference in depression. In recent years, investigators have moved toward more integrated models, taking a transactional, developmental approach. Transactional models are appropriate because it is clear that depression impairs social and occupational functioning, and thus can have a major impact on an individual's environment. Developmental models are appropriate because age groups differ markedly in the gender difference in depression. Girls are no more likely than boys to evidence depression in childhood, but by about age 13, girls' rates of depression begin to increase sharply, whereas boys' rates of depression remain low, and may even decrease. By late adolescence, girls are twice as likely as boys to be depressed, and this gender ratio remains more or less the same throughout adulthood. The absolute rates of depression in women and men vary substantially across the life span, however.

In this review, I focus on two themes in recent research. First, because women have less power and status than men in most societies, they experience certain traumas, particularly sexual abuse, more often than men. They also experience more chronic strains, such as poverty, harassment, lack of respect, and constrained choices. Second, even when women and men experience the same stressors, women may be more likely than men to develop depression because of gender differences in biological responses to stressors, self-concepts, or coping styles.

Frequent stressful experiences and reactivity to stress are likely to have reciprocal effects on each other. Stressful experiences can sensitize both biological and psychological systems to future stress, making it more likely that individuals will react with depression. In turn, reactivity to stress is associated with impaired problem solving, and, as a result, with the accumulation or generation of new stressors, which may contribute to more depression.

\section{STRESSFUL LIFE EVENTS}

Women's lack of social power makes them more vulnerable than men to specific major traumas, particularly sexual abuse. Traumas may contribute directly to depression, by making women feel they are helpless to control their lives, and may also contribute indirectly, by increasing women's reactivity to stress. Women's social roles also carry a number of chronic strains that might contribute directly or indirectly to depression. Major changes in the frequency of traumatic events and in social roles coincide with the emergence of gender differences in depression in adolescence, and may help to explain this emergence.

\section{Victimization}

Women are the victims of sexual assault-defined as being pressured or forced into unwanted sexual contact-at least twice as often as men, and people with a history of sexual assault have increased rates of depression (see Weiss, Longhurst, \& Mazure, 1999). Sexual assault during childhood has been more consistently linked with the gender difference in depression than sexual assault that first occurs during adulthood. Estimates of the prevalence of childhood sexual assault range widely. Cutler and I reviewed the most methodologically 
sound studies including both male and female participants and found rates of childhood sexual assault between 7 and 19\% for females and between 3 and $7 \%$ for males (Cutler \& Nolen-Hoeksema, 1991). We estimated that, in turn, as much as $35 \%$ of the gender difference in adult depression could be accounted for by the higher incidence of assault of girls relative to boys. A few studies have examined whether depression might be an antecedent rather than a consequence of sexual assault. Depression does appear to increase risk for sexual assault in women and men, but sexual assault significantly increases risk for first or new onsets of depression.

Childhood sexual assault may increase risk for depression throughout the life span because abuse experiences negatively alter biological and psychological responses to stress (Weiss et al., 1999). Children and adolescents who have been abused, particularly those who have been repeatedly abused over an extended period of time, tend to have poorly regulated biological response to stress. Abuse experiences can also negatively alter children's and adolescents' perspectives on themselves and others, contributing to their vulnerability to depression (Zahn-Waxler, 2000).

\section{Chronic Strains}

Women face a number of chronic burdens in everyday life as a result of their social status and roles relative to men, and these strains could contribute to their higher rates of depression (see Nolen-Hoeksema, 1990). Women make less money than men, and are much more likely than men to live in poverty. Women are more likely than men to be sexually harassed on the job. Women often have full-time paid jobs and also do nearly all the child care and domestic work of the home. In addition, women are increasingly "sandwiched" between caring for young children and caring for sick and elderly family members. This role overload is said to contribute to a sense of "burn out" and general distress, including depressive symptoms, in women.

In the context of heterosexual relationships, some women face inequities in the distribution of power over important decisions that must be made, such as the decision to move to a new city, or the decision to buy an expensive item such as a car (Nolen-Hoeksema, Larson, \& Grayson, 1999). Even when they voice their opinions, women may feel these opinions are not taken seriously, or that their viewpoints on important issues are not respected and affirmed by their partners. My colleagues and I measured chronic strain by grouping inequities in workload and heterosexual relationships into a single variable, and found that this variable predicted increases in depression over time, and partially accounted for the gender difference in depression (Nolen-Hoeksema et al., 1999). Depression also contributed to increased chronic strain over time, probably because it was associated with reductions in perceptions of control and effective problem solving.

\section{Gender Intensification in Adolescence}

Social pressure to conform to gender roles is thought to increase dramatically as children move through puberty. For girls, this may mean a reduction in their opportunities and choices, either real or perceived. According to adolescents' own reports, parents restrict girls' more than boys' behaviors and have lower expectations for girls' than for boys' competencies and achievements. Girls also feel that if they pursue male-stereotyped activities and preferences, such as interests in math and science or in competitive sports, they are rejected by their peers. For many girls, especially white girls, popularity and social acceptance become narrowly oriented around appearance.

This narrowing of acceptable behavior for girls in early adolescence may contribute to the increase in depression in girls at this time, although this popular theory has been the focus of remarkably little empirical research (NolenHoeksema \& Girgus, 1994). There is substantial evidence that excessive concern about appearance is negatively associated with wellbeing in girls, but these findings may apply primarily to white girls. In addition, very little research has examined whether appearance concerns and gender roles are risk factors for depression or only correlates.

\section{REACTIVITY TO STRESS}

Even when women and men are confronted with similar stressors, women may be more vulnerable than men to developing depression and related anxiety disorders such as posttraumatic stress disorder (Breslau, Davis, Andreski, Peterson, \& Schultz, 1997). Women's greater reactivity compared with men's has been attributed to gender differences in biological responses, self-concepts, and coping styles.

\section{Biological Responses to Stress}

For many years, the biological explanations for women's greater vulnerability to depression focused on the direct effects of the ovarian hormones (especially estrogen and progesterone) on women's moods. This literature is too large and com- 
plicated to review here (but see Nolen-Hoeksema, 1990, 1995). Simply put, despite widespread popular belief that women are more prone to depression than men because of direct negative effects of estrogen or progesterone on mood, there is little consistent scientific evidence to support this belief. Although some women do become depressed during periods of hormonal change, including puberty, the premenstrual period of the menstrual cycle, menopause, and the postpartum period, it is unclear that these depressions are due to the direct effects of hormonal changes on mood, or that depressions during these periods of women's lives account for the gender differences in rates of depression.

More recent biological research has focused not on direct effects of ovarian hormones on moods, but on the moderating effects of hormones, particularly adrenal hormones, on responses to stress. The hypothalamic-pituitary-adrenal (HPA) axis plays a major role in regulating stress responses, in part by regulating levels of a number of hormones, including cortisol, which is released by the adrenal glands in response to chemicals secreted by the brain's hypothalamus and then the pituitary. In turn, cortisol levels can affect other biochemicals known to influence moods. People with major depressive disorder often show elevated cortisol responses to stress, indicating dysregulation of the HPA response.

An intriguing hypothesis is that women are more likely than men to have a dysregulated HPA response to stress, which makes them more likely to develop depression in response to stress (Weiss et al., 1999). Women may be more likely to have a dysregulated HPA response because they are more likely to have suffered traumatic events, which are known to contribute to HPA dysregulation. In addition, ovarian hormones modulate regulation of the HPA axis (Young \& Korszun, 1999). Some women may have depressions during periods of rapid change in levels of ovarian hormones (the postpartum period, premenstrual period, menopause, and puberty) because hormonal changes trigger dysregulation of the stress response, making these women more vulnerable to depression, particularly when they are confronted with stress. The causal relationship between HPA axis regulation and the gender difference in depression has not been established but is likely to be a major focus of future research.

\section{Self-Concept}

Although the idea that girls have more negative self-concepts than boys is a mainstay of the pop-psychology literature, empirical studies testing this hypothesis have produced mixed results (Nolen-Hoeksema \& Girgus, 1994). Several studies have found no gender differences in self-esteem, self-concept, or dysfunctional attitudes. Those studies that do find gender differences, however, tend to show that girls have poorer self-concepts than boys. Again, negative self-concepts could contribute directly to depression, and could interact with stressors to contribute to depression. Negative self-concept has been shown to predict increases in depression in some studies of children (Nolen-Hoeksema \& Girgus, 1994).

One consistent difference in males' and females' self-concepts concerns interpersonal orientation, the tendency to be concerned with the status of one's relationships and the opinions others hold of oneself. Even in childhood, girls appear more interpersonally oriented than boys, and this gender difference increases in adolescence (Zahn-Waxler, 2000). When interpersonal orientation leads girls and women to subordinate their own needs and desires completely to those of others, they become excessively dependent on the good graces of others (Cyranowski, Frank, Young, \& Shear, 2000). They may then be at high risk for depression when conflicts arise in relationships, or relationships end. Several recent studies have shown that girls and women are more likely than boys and men to develop depression in response to interpersonal stressors. Because depression can also interfere with interpersonal functioning, an important topic for future research is whether the gender difference in depression is a consequence or cause of gender differences in interpersonal strain.

\section{Coping Styles}

By adolescence, girls appear to be more likely than boys to respond to stress and distress with rumination-focusing inward on feelings of distress and personal concerns rather than taking action to relieve their distress. This gender difference in rumination then is maintained throughout adulthood. Several longitudinal and experimental studies have shown that people who ruminate in response to stress are at increased risk to develop depressive symptoms and depressive disorders over time (Nolen-Hoeksema et al., 1999). In turn, the gender difference in rumination at least partially accounts for the gender difference in depression. Rumination may not only contribute directly to depression, but may also contribute indirectly by impairing problem solving, and thus preventing women from taking action to overcome the stressors they face.

\section{AN INTEGRATIVE MODEL}

Women suffer certain stressors more often than men and may be 
more vulnerable to develop depression in response to stress because of a number of factors. Both stress experiences and stress reactivity contribute directly to women's greater rates of depression compared with men. Stress experiences and stress reactivity also feed on each other, however. The more stress women suffer, the more hyperresponsive they may be to stress, both biologically and psychologically. This hyperresponsiveness may undermine women's ability to control their environments and overcome their stress, leading to even more stress in the future. In addition, depression contributes directly to more stressful experiences, by interfering with occupational and social functioning, and to vulnerability to stress, by inciting rumination, robbing the individual of any sense of mastery she did have, and possibly sensitizing the biological systems involved in the stress response.

Important advances will be made in explaining the gender difference in depression as we understand better the reciprocal effects of biological, social, and psychological systems on each other. Key developmental transitions, particularly the early adolescent years, are natural laboratories for observing the establishment of these processes, because so much changes during these transitions, and these transitions are times of increased risk.

Additional questions for future research include how culture and ethnicity affect the gender differ- ence in depression. The gender difference is found across most cultures and ethnicities, but its size varies considerably, as do the absolute percentages of depressed women and men. The processes contributing to the gender difference in depression may also vary across cultures and ethnicities.

Understanding the gender difference in depression is important for at least two reasons. First, women's high rates of depression exact tremendous costs in quality of life and productivity, for women themselves and their families. Second, understanding the gender difference in depression will help us to understand the causes of depression in general. In this way, gender provides a valuable lens through which to examine basic human processes in psychopathology.

\section{Recommended Reading}

Cyranowski, J.M., Frank, E., Young, E., \& Shear, K. (2000). (See References)

Nolen-Hoeksema, S. (1990). (See References)

Nolen-Hoeksema, S., \& Girgus, J.S. (1994). (See References)

Nolen-Hoeksema, S., Larson, J., \& Grayson, C. (1999). (See References)

Young, E., \& Korszun, A. (1999). (See References)

\section{Note}

1. Address correspondence to Susan Nolen-Hoeksema, Department of Psychology, University of Michigan, $525 \mathrm{E}$. University Ave., Ann Arbor, MI 48109; e-mail: nolen@umich.edu.

\section{References}

Breslau, N., Davis, G.C., Andreski, P., Peterson, E.L., \& Schultz, L. (1997). Sex differences in posttraumatic stress disorder. Archives of General Psychiatry, 54, 1044-1048.

Cutler, S., \& Nolen-Hoeksema, S. (1991). Accounting for sex differences in depression through female victimization: Childhood sexual abuse. Sex Roles, 24, 425-438.

Cyranowski, J.M., Frank, E., Young, E., \& Shear, K. (2000). Adolescent onset of the gender difference in lifetime rates of major depression. Ar chives of General Psychiatry, 57, 21-27.

Kessler, R.C., McGonagle, K.A., Swartz, M., Blazer, D.G., \& Nelson, C.B. (1993). Sex and depression in the National Comorbidity Survey I: Lifetime prevalence, chronicity, and recurrence. Journal of Affective Disorders, 29, 85-96.

Nolen-Hoeksema, S. (1990). Sex differences in depression. Stanford, CA: Stanford University Press.

Nolen-Hoeksema, S. (1995). Gender differences in coping with depression across the lifespan. Depression, 3, 81-90.

Nolen-Hoeksema, S., \& Girgus, J.S. (1994). The emergence of gender differences in depression in adolescence. Psychological Bulletin, 115, 424-443.

Nolen-Hoeksema, S., Larson, J., \& Grayson, C. (1999). Explaining the gender difference in depression. Journal of Personality and Social Psychology, 77, 1061-1072.

Weiss, E.L., Longhurst, J.G., \& Mazure, C.M (1999). Childhood sexual abuse as a risk factor for depression in women: Psychosocial and neurobiological correlates. American Journal of Psychiatry, 156, 816-828.

Weissman, M.M., Bland, R.C., Canino, G.J., Faravelli, C., Greenwald, S., Hwu, H.-G., Joyce, P.R. Karam, E.G., Lee, C.-K., Lellouch, J., Lepine, J.P., Newman, S.C., Rubio-Stipc, M., Wells, E. Wickramaratne, P.J., Wittchen, H.-U., \& Yeh, E.K. (1996). Cross-national epidemiology of major depression and bipolar disorder. Journal of the American Medical Association, 276, 293-299.

Young, E., \& Korszun, A. (1999). Women, stress, and depression: Sex differences in hypothalamic-pituitary-adrenal axis regulation. In E. Leibenluft (Ed.), Gender differences in mood and anxiety disorders: From bench to bedside (pp. 31-52). Washington, DC: American Psychiatric Press.

Zahn-Waxler, C. (2000). The development of empathy, guilt, and internalization of distress: Implications for gender differences in internalizing and externalizing problems. In R. Davidson (Ed.), Wisconsin Symposium on Emotion: Vol. 1. Anxiety, depression, and emotion (pp. 222-265). Oxford, England: Oxford University Press. 